\title{
Sacrificial bonds heal bone
}

John Currey

The toughness of bone is usually attributed to its collagen, but how does it work? New evidence shows that molecular bonds can temporarily sacrifice themselves to absorb impacts.

$\mathrm{B}$ one's 'toughness'

its ability to absorb impacts and resist the weakening effects of small scratches and holes is almost as vital to its function as its 'stiffness'. The stiffness of bone, which provides skeletal rigidity, is reasonably well understood: it is produced by tiny mineral crystals that permeate its organic material. Stiffness varies considerably according to the relative amounts of mineral, organic material and water. However, the origin of toughness is still somewhat mysterious. In an article on page 773 of this issue, Thompson and colleagues ${ }^{1}$ suggest that collagen the main organic constituent of bone has 'sacrificial bonds' that are broken upon loading without significantly harming the bone. Bone's toughness comes from the mechanical work required to overcome these bonds. Some of these sacrificial bonds reform when the force is removed, allowing bone to repair itself to some extent.

A tough material such as bone requires much mechanical work before it fractures. Bone's force-extension curve (Fig. 1) gives a graphical representation of this toughness. Up to the 'yield' point — the range in which bone normally functions - a significant amount of force is required to cause extension. After yield, the curve flattens out, so elongation requires very little extra force. Atomic force microscopy was originally developed to produce highly detailed images of surfaces, but it can also be used to produce force-extension curves for single protein molecules, such as collagen. The protein is stretched by a diamond on the end of a cantilever so that the force required, and the distance that the protein is stretched, can be measured. In addition, the diamond can be pressed into the surface of hard materials such as bone, and the force of the elastic recoil can be measured to determine the mechanical properties at that point in the surface ${ }^{2}$.

Thompson and colleagues ${ }^{1}$ first tried this technique on collagen molecules spread on a glass sheet. They found that the forceextension curve, instead of rising smoothly, was saw-toothed with periodic drops. This meant that the collagen kept on extending, requiring significant work in the process, but was never subjected to forces large enough to cause rupture. They also found that less work was needed to extend the collagen on subsequent stretches but that the work needed gradually recovered with time, although never completely. Thompson and colleagues attributed these saw-toothed patterns to the breaking of sacrificial bonds - bond breakage causing a sudden decrease in the force required - which gradually reformed when the force was removed. The experiment was repeated on molecules exposed on the surface of polished bone, and similar effects were found. In particular, the time course of the recovery in work required was almost the same. These sawtoothed patterns are not visible in the analogous region of the force-extension curve for bone (the flat part of the curve) owing to the inability to obtain such high resolution on whole specimens.

A clue to the nature of the sacrificial bonds came when the authors soaked the collagen or bone in different solutions. More work was required on subsequent stretches when a solution containing divalent calcium ions was used than when the solution contained monovalent sodium ions. Indeed, the specimens showed little saw-tooth behaviour in the sodium-containing solution. As divalent, but not monovalent, ions can link molecules together, this suggests that some kind of ionic bond between charged residues in collagen might be responsible for reconnecting the individual collagen molecules to each other. Furthermore, previous work from this group has shown similar results for the organic material between the little plates of calcium carbonate in mother of pearl ${ }^{3}$, so it could be that this method of toughening is widespread among calcified tissues.

These findings are potentially important for understanding the postyield behaviour of bone (Fig. 1). Nevertheless, there is still a considerable element of speculation in all this. Thompson et al. cannot be sure that they are stretching collagen molecules in their experiments with bone, although it is likely that they are, given the similar saw-toothed curves. In addition, they have no means of knowing whether they are stretching a single molecule or more than one, which might confound the interpretation of the saw-toothed curve. Finally, it is curious that they find very similar amounts of work to be required when pulling on collagen and bone. In bone, collagen is bound rather tightly to the mineral, so the ability to stretch free collagen molecules seems unlikely.

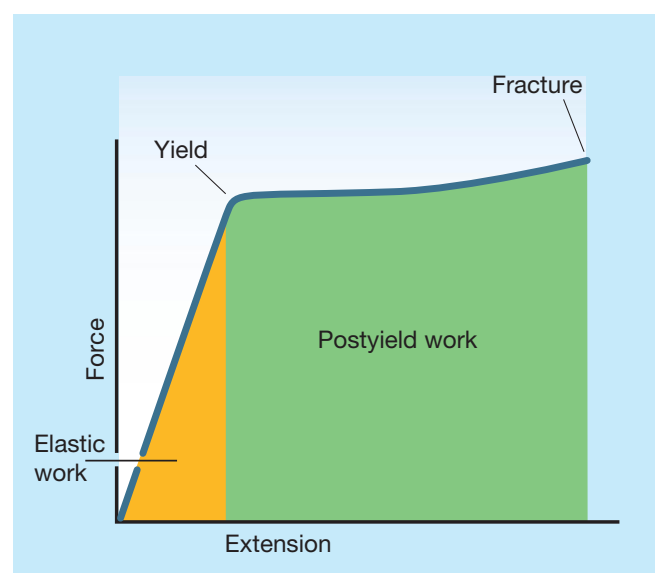

Figure 1 Force-extension curve for bone. Bone is elastic up to the yield point and so it can return to its original state when unloaded. This is the range in which bone normally functions. At yield, and after, damage occurs such that bone remains distorted upon unloading and is less stiff than before; that is, it requires a smaller force to produce the same extension. Thompson and colleagues ${ }^{1}$ suggest that this reduced stiffness is due to the temporary breakage of sacrificial bonds within the bone's collagen molecules, which begins after the bone has passed its yield point.

Some researchers think that the postyield behaviour of bone is caused by extensive microcracking ${ }^{4,5}$. When bone undergoes postyield extensions, innumerable microcracks begin, spread slightly, but then come to a halt. So this flat part of the curve is thought to be caused by the reduced stiffness induced by the microcracks. In other words, bone is damaged by these microcracks, but remains coherent until fracture. Microcrack-induced elasticity can adequately explain the behaviour of bone in qualitative terms. However, at present it is impossible to assign numbers to this effect and the qualitative explanation might not, in the end, give the right quantitative answers.

Others, including of course Thompson et al., think that the postyield behaviour of bone is best considered at a more molecular level than microcracking — at the level of bonding of mineral crystals to the organic matrix and of molecular interactions within collagen itself. Whether this latter mechanism is involved in preventing the spread of microcracks is unknown; if it is, it would be fascinating to see the two theories reconciled.

John Currey is in the Department of Biology,

University of York, York YO10 5YW, UK.

e-mail:jdc1@york.ac.uk

1. Thompson, J. B. et al. Nature 414, 773-776 (2001).

2. Oliver, W. C. \& Pharr, G. M. J. Materials Res. 7, 1564-1583 (1992).

3. Smith, B. L. et al. Nature 399, 761-763 (1996).

4. Zioupos, P. \& Currey, J. D. J. Materials Sci. 29, 978-986 (1994).

5. Vashishth, D., Tanner, K. E. \& Bonfield, W. J. Biomech. 33, 1169-1174 (2000). 